\title{
Revisión
}

\section{Guía clínica del carcinoma urotelial no músculo invasivo de la Asociación Europea de Urologia*}

\author{
Marko Babjuk ${ }^{\mathrm{a}}$, Willem Oosterlinck ${ }^{\mathrm{b}}$, Richard Sylvester ${ }^{\mathrm{c}}$, Eero Kaasinen ${ }^{\mathrm{d}}$, Andreas Böhle , \\ Juan Palou-Redorta ${ }^{\mathrm{f}}$
} aServicio de Urología, Hospital General de Enseñanza, Primera Facultad de Medicina, Universidad de Carlos,
Praga, República Checa

${ }^{b}$ Servicio de Urología, Hospital Universitario de Gante, Gante, Bélgica

${ }^{c}$ Oficina central de la EORTC, Bruselas, Bélgica

dServicio de Urología, Hyvinkää, Finlandia

eservicio de Urología, Bad Schwartau, Alemania

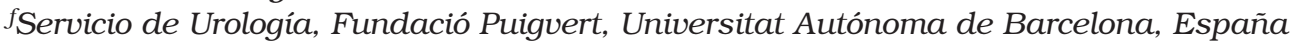

Traducción del artículo Eur Urol 2008; 54: 303-14: Saturnino Luján Marco

Servicio de Urología. Hospital Universitario La Fe. Valencia, España

\section{Resumen}

Contexto y objetivos: Presentar una puesta al día de la versión del 2008 de la guía clínica de la Asociación Europea de Urología (EUA) sobre el cáncer vesical no músculo invasivo.

Evidencia adquirida: Se ha realizado una revisión sistemática de la literatura reciente acerca del diagnóstico y tratamiento del cáncer vesical no músculo invasivo. Las guías clínicas fueron puestas al día y se asignó un nivel de evidencia así como un grado de recomendación. Evidencia sintetizada: El diagnóstico del cáncer vesical depende de la cistoscopia y de los hallazgos histológicos del tejido resecado. Una correcta y completa resección transuretral (RTU) es esencial en el pronóstico del paciente. Cuando la primera resección es incompleta o cuando se diagnostica un tumor de alto grado o T1, se debe realizar una segunda resección a las 2-6 semanas.

El riesgo a corto y a largo plazo tanto de la recidiva como de la progresión serán calculados de manera individual a través de tablas de riesgo y sistemas de puntuación. La estratificación de los pacientes en grupos de bajo, intermedio y alto riesgo (separando la recidiva y la progresión), supone la piedra angular para indicar un tratamiento adyuvante. Es altamente recomendable en pacientes de bajo riesgo de recidiva y progresión, una instilación inmediata de quimioterapia. En aquellos que tienen riesgo intermedio o alto de recidiva y un riesgo intermedio de progresión, se debe administrar una instilación inmediata de quimioterapia seguido de instilaciones periódicas de quimioterapia o un mínimo de un año con Bacilo de Calmette-Guerin (BCG). En pacientes con alto riesgo de progresión tumoral, tras una administración inmediata de quimioterapia, está indicado BCG intravesical como mínimo durante un año. Una cistectomía inmediata debería ser ofrecida a pacientes de altísimo riesgo y en pacientes en los que ha fallado la BCG. La versión extensa de las guías clínicas está disponible en www.uroweb.org

Conclusiones: Esta guía clínica de la EUA presenta una información actualizada sobre el diagnóstico y tratamiento del cáncer vesical además de ofrecer recientes hallazgos con el fin de aplicarlos a la práctica clínica diaria.

Palabras clave: Bacilo de Calmette-Guerin (BCG). Cáncer de vejiga. Cistectomía. Cistoscopia. Diagnóstico. Guías clínicas de la EUA. Seguimiento. Quimioterapia intravesical. Pronóstico. Resección transuretral (RTU).

\section{EAU Guidelines on Non-Muscle-Carcinoma of the Bladder}

\section{Abstract}

Context and objective: To present the updated version of 2008 European Association of Urology (EAU) guidelines on non-muscle-invasive bladder cancer.

Evidence acquisition: A systematic review of the recent literature on the diagnosis and treatment of non-muscle-invasive bladder cancer was performed. The guidelines were updated and the level of evidence and grade of recommendation were assigned.

Evidence synthesis: The diagnosis of bladder cancer depends on cystoscopy and histologic evaluation of the resected tissue. A complete and correct transurethral resection (TUR) is essential for the prognosis of the patient. When the initial resection is incomplete or when a high-grade or T1 tumour is detected, a second TUR within 2-6 wk should be performed.

The short- and long-term risks of both recurrence and progression may be estimated for individual patients using the scoring system and risk tables. The stratification of patients to low, intermediate, and high-risk groups-separately for recurrence and progression-represents the cornerstone for indication of adjuvant treatment. In patients at low risk of tumour recurrence and progression, one immediate instillation of chemotherapy is strongly recommended. In those at an intermediate or high risk of recurrence and an intermediate risk of progression, one immediate instillation of chemotherapy should be followed by further instillations of chemotherapy or a minimum of 1 yr of bacillus Calmette-Guerin (BCG). In patients at high risk of tumour progression, after an immediate instillation of chemotherapy, intravesical BCG for at least $1 \mathrm{yr}$ is indicated. Immediate cystectomy may be offered to the highest risk patients and in patients with BCG failure. The long version of the guidelines is available on www.uroweb.org.

Conclusions: These EAU guidelines present the updated information about the diagnosis and treatment of non-muscle-invasive bladder cancer and offer the recent findings for the routine clinical application.

Keywords: Bacillus Calmette-Guerin (BCG). Bladder cancer. Cystectomy. Cystoscopy. Diagnosis. EAU Guidelines. Follow-up. Intravesical chemotherapy. Prognosis Transurethral resection (TUR).

(*)La traducción de este artículo se ha llevado a cabo con el permiso de la Asociación Europea de Urología. 


\section{Antecedentes}

La primera guía clínica de la Asociación Europea de Urología (EAU) sobre el cáncer vesical fue publicada en $2002^{1}$. Desde entonces, la versión extensa de la guía clínica ha sido puesta al día continuamente, con la última versión disponible en www.uroweb.org. Esta visión general representa una versión actualizada de la guía clínica de la EAU del cáncer vesical no músculo invasivo del 2008.

\section{Epidemiología}

El carcinoma de vejiga es la más común de las patologías malignas del tracto urinario. En Europa, la incidencia más alta (tasa estandarizada por edad) aparece en Europa Occidental (23,6 en hombres y 5,4 en mujeres) y en las zonas del sur $(27,1$ en hombres y 4,1 en mujeres) seguido por el norte de Europa (16,9 en hombres y 4,9 en mujeres). La incidencia más baja aparece en los países de Europa Oriental ( 14,7 en hombres y 2,2 en mujeres respectivamente) $)^{2}$

Aproximadamente $75-85 \%$ de los pacientes con cáncer de vejiga presentan enfermedad confinada a la mucosa (estadio Ta, CIS) o submucosa (estadio T1).

\section{Clasificación}

La clasificación del 2002 de Tumor, Nódulo, Metástasis (TNM) aprobada por la Unión Internacional Contra el Cáncer (UICC), ha sido ampliamente aceptada y es la usada en esta guía clínica (Tabla 1$)^{3}$.

La nueva clasificación para el grado del tumor urotelial no músculo invasivo fue propuesta por la Organización Mundial para la Salud (OMS) y la Sociedad Internacional de Patología Urológica (ISUP) y publicada por la OMS en 2004 (Tabla 2) ${ }^{4}$. Se diferencian entre Neoplasia Papilar Urotelial de Bajo Potencial de Malignidad (NPUBPM) y carcinomas uroteliales de alto y bajo grado.

La NPUBPM son lesiones que no tienen rasgos citológicos de malignidad pero muestran células uroteliales normales dentro de una configuración papilar. Estas lesiones tienen un riesgo insignificante de progresar, pero si mantienen una tendencia a la recidiva. El grado intermedio (grado 2), el cual había sido objeto de controversia en la clasificación de la OMS de 1973, ha sido eliminado.

Hasta que la clasificación del 2004 de la OMS haya sido validada en más ensayos clínicos, los tumores deben ser clasificados usando tanto la clasificación de la OMS del 1973 como la del 2004 (Tabla 2).

Tabla 1. Clasificación 2002 TNM cáncer de vejiga

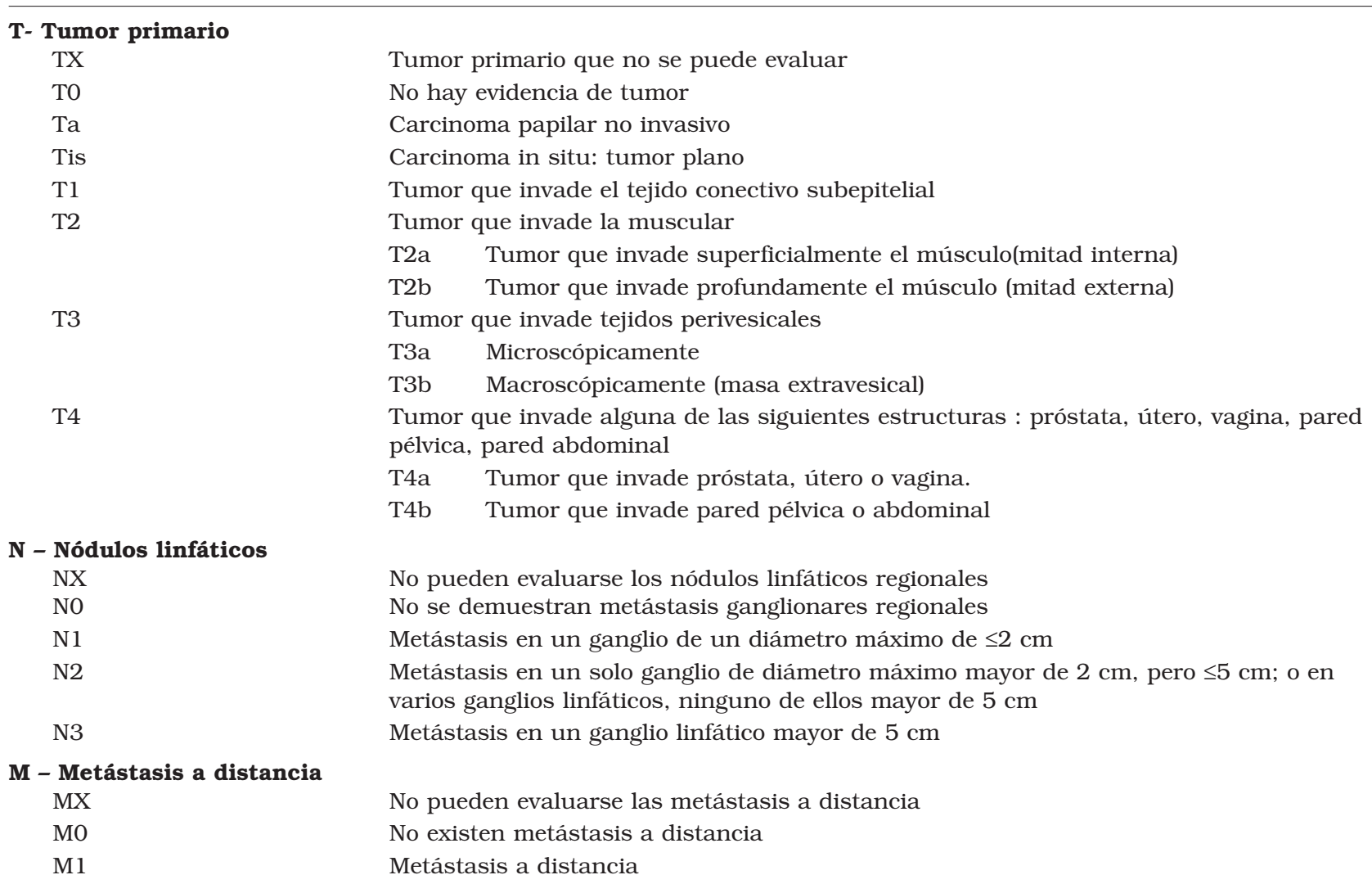


Tabla 2. Grados de la OMS en 1973 y 2004

\section{3}

Papiloma urotelial

Grado 1: bien diferenciado

Grado 2: moderadamente diferenciado

Grado 3: pobremente diferenciado

\section{4}

Papiloma urotelial

Neoplasia Papilar Urotelial de Bajo Potencial de

Malignidad (NPUBPM)

Carcinoma papilar urotelial de bajo grado

Carcinoma papilar urotelial de alto grado

A pesar de unos criterios bien definidos, hay una importante variabilidad interobservador en la clasificación de la displasia y carcinoma in situ (CIS,Tis), estadio $\mathrm{T} 1$ frente a tumores Ta y grado de los tumores $^{5}$. Como consecuencia de ello nosotros recomendamos que los urólogos revisen los hallazgos histológicos junto con los patólogos.

\section{Factores de riesgo}

Los urólogos deben de conocer los tipos de exposiciones laborales que están relacionadas con los carcinógenos uroteliales $^{6}$. Los primeros en identificarse fueron las aminas aromáticas. Como grupos de riesgos se incluyen trabajadores de las siguientes industrias: imprenta, hierro y procesado de aluminio, pinturas industriales, gas y alquitrán (nivel de evidencia: 3).

Otro factor de riesgo importante es el tabaco, el cual triplica el riesgo de desarrollar cáncer de vejiga y que conduce a una mayor mortalidad $^{7}$ (nivel de evidencia: 3).

\section{Diagnóstico}

La hematuria es el hallazgo más común de los tumores vesicales no músculo invasivo. $\mathrm{El}$ aumento de la frecuencia miccional, disuria o urgencia podrían ser sintomas de CIS.

\subsection{Diagnóstico por imagen}

Mediante la urografía intravenosa (UIV) los tumores de gran tamaño pueden aparecer como defectos de replección. También puede utilizarse para detectar defectos de replección en el tracto urinario superior o hidronefrosis, los cuales pueden indicar la presencia de un tumor ureteral. La necesidad de utilizar de manera rutinaria la UIV está ahora cuestionada por la baja incidencia de hallaz- gos significativos ${ }^{8,9}$ (nivel de evidencia: 3). La incidencia de tumores simultáneos en el tracto urinario superior es baja (1,8\%), pero se incrementa un 7,5\% en tumores localizados en el trígono ${ }^{8}$. El riesgo de recidiva tumoral sobre el tracto urinario superior durante el seguimiento se incrementa en los tumores múltiples y de alto grado ${ }^{9}$.

En muchos centros, el Uro-TAC se usa como una alternativa a la UIV convencional.

La ecografía transabdominal permite caracterizar las masas renales, detectar hidronefrosis, y visualizar lesiones de la pared vesical. Combinado con la radiografía simple abdominal, puede ser tan adecuado como la UIV en el diagnóstico de la hematuria (nivel de evidencia: 3).

\subsection{Citología urinaria}

El examen de orina tras micción o muestra urinaria de células tumorales exfoliadas obtenidas tras lavado vesical, muestra una alta sensibilidad para tumores de alto grado, pero baja sensibilidad en tumores de bajo grado (nivel de evidencia: $2 a)^{10}$. Por tanto será útil cuando sospeche la presencia de CIS o cáncer de alto grado; sin embargo si el resultado de la citología es negativo no podemos excluir la presencia de cáncer de bajo grado.

La positividad de una citología puede indicar la presencia de tumor urotelial en cualquier segmento del tracto urinario. La interpretación de la citología es observador dependiente ${ }^{11}$. La interpretación puede alterarse en caso de una escasa muestra celular, infección del tracto urinario, litiasis, o tras instilaciones vesicales. En manos experimentadas la especificidad sobrepasa el $90 \%{ }^{10}$ (nivel de evidencia:2a). La citología debe interpretarse con orina fresca y con una adecuada fijación. La orina de la mañana no es adecuada ya que suele presentar citolisis.

\subsection{Tests moleculares de orina}

Disponemos de diferentes test para la detección de marcadores solubles o celulares en orina ${ }^{10}$. Muchos de estos test tienen una mejor sensibilidad para detectar cáncer vesical que la citología, pero su especificidad es baja (nivel de evidencia: 2a). Permanece sin aclarar cuál de estos test nos ofrece una información adicional que nos sea útil para la detección y el manejo del tumor de vejiga no músculo invasivo ${ }^{10}$. Por otro lado, debemos considerar el coste adicional de los mismos. 


\subsection{Cistoscopia}

El diagnóstico del cáncer de vejiga depende en último término del examen bajo cistoscopia y la evaluación histológica del tejido resecado.

En general, la cistoscopia se realizada primero en las consultas, mediante instrumentos flexibles. Si el tumor vesical ha sido visualizado previamente en estudios de imagen, el diagnóstico con cistoscopia puede ser omitido. Es necesaria una descripción cuidadosa de los hallazgos. Ésta debe incluir la localización, tamaño, número, y la apariencia (papilar o sólido) de los tumores así como anormalidades de la mucosa.

\subsection{Resección transuretral (RTU)}

El fin de la RTU en los tumores TaT1 es realizar un correcto diagnóstico y eliminar todas las lesiones visibles.

Los tumores pequeños (menores de $1 \mathrm{~cm}$ ) pueden resecarse en bloque, y la muestra debe tener la porción inferior de la pared vesical. Algunos expertos creen que una resección profunda no es necesaria en lesiones pequeñas, con apariencia de bajo grado e historia previa de tumores TaG1.

Los tumores grandes deberían ser resecados separadamente en fracciones, las cuales deben incluir la parte exofítica del tumor, la parte inferior de la pared vesical con el músculo detrusor, y los bordes del área resecada. Las muestras de las diferentes fracciones deberían remitidas al patólogo en frascos separados y referenciados. La cauterización debería ser evitada en la medida de lo posible durante la resección para evitar la destrucción de los tejidos.

El informe patológico debería especificar el grado de la lesión, y la profundidad de la invasión tumoral en la pared vesical e informar si la lámina propia y el músculo están presentes en la muestra ${ }^{12}$.

Una RTU completa y correcta es esencial para el pronóstico del paciente ${ }^{13}$.

\subsection{Biopsia vesical y de la uretra prostática}

Los tumores vesicales suelen ser multifocales. Por otra parte TaT1 pueden asociar CIS o displasia. Estas lesiones pueden presentarse como lesiones aterciopeladas, áreas eritematosas indistinguibles de lesiones inflamatorias o pueden no ser visibles.

Las biopsias de la mucosa de apariencia normal en pacientes con tumor TaT1, llamadas biopsias aleatorias o biopsias selectivas de la mucosa, no están recomendadas de manera rutinaria. La probabilidad de detectar CIS en los tumores de bajo riesgo es extremadamente baja (por debajo del 2\%), y la elección de una terapia adyuvante intravesical no influye por los resultados de la biopsia ${ }^{14}$ (nivel de evidencia: 2a). La biopsia con pinza fría de la mucosa con apariencia normal debería realizarse cuando la citología es positiva o cuando un tumor exofítico es de apariencia no papilar. Cuando aparecen áreas anormales de urotelio se recomienda tomar biopsias con pinza fría o biopsias con resector. El material obtenido en las biopsias aleatorias o bien directamente debería ser enviado para la valoración por el patólogo en frascos separados.

En varones con tumores TaT1, se ha descrito la afectación de la uretra prostática y los ductus prostáticos. Aunque el riesgo exacto no es conocido, si que parece que es mayor en los tumores que se localizan en el trígono o en el cuello vesical, en presencia de CIS, y en la multiplicidad tumoral ${ }^{15,16}$ (nivel de evidencia: 3). En estos casos y cuando la citología es positiva sin evidencia de tumor vesical o cuando son visibles anormalidades en la uretra prostática, debemos considerar realizarla.

\subsection{Cistoscopia fluorescente}

El uso de luz blanca nos puede llevar a perder lesiones que están presentes pero no visibles.

La cistoscopia fluorescente se realiza usando una luz ultravioleta tras la instilación de un fotosensibilizador o su precursor, generalmente ácido 5 aminolevulínico (5-ALA) o hexalaminolevulinato (HAL). Se ha comprobado que la biopsia y resección guiada por fluorescencia es más sensible que los procedimientos habituales en la detección de tumores malignos, particularmente tipo CIS $^{17}$ (nivel de evidencia: 2 a). Sin embargo puede haber falsos positivos, en procesos inflamatorios, RTU reciente o por instilaciones vesicales.

El beneficio de la RTU guiada por fluorescencia para la supervivencia libre de recidiva se ha demostrado en varios ensayos clínicos aleatorizados ${ }^{18}$, pero su valor definitivo en la mejora de los resultados de progresión o supervivencia de los pacientes aun no se ha demostrado. Debemos considerar el coste adicional del equipamiento necesario.

\subsection{Segunda resección}

Se ha demostrado que existe un riesgo significativo de tumor residual tras la RTU de los tumores 
TaT1 ${ }^{13,19}$ (nivel de evidencia :1). Por otra parte los tumores pueden ser infradiagnosticados en la resección inicial.

Debemos considerar una segunda RTU si existe la sospecha de una primera resección incompleta, cuando se trata de tumores múltiples o de gran tamaño o cuando el patólogo no informa de la presencia de tejido muscular en la muestra. Además, deberá realizarse cuando en la resección inicial aparece un tumor no músculo invasivo de alto grado o $\mathrm{T} 1$.

Se ha demostrado que una segunda RTU puede aumentar la supervivencia libre de recidiva y progresión ${ }^{20}$ (nivel de evidencia: 2 a). Muchos autores recomiendan esta resección a las 2-6 semanas de la primera RTU. Esta resección deberá incluir la localización del tumor primario. En la Tabla 3 se encuentra el resumen de las recomendaciones para el diagnóstico de cáncer de vejiga no músculo-invasivo.

\section{Predicción de la recidiva y progresión}

La vía clásica para categorizar a los pacientes con tumores TaT1 es dividirlos en grupos de riesgo basados en factores pronósticos a partir de análisis multivariantes. Se ha desarrollado un sistema de puntuación y unas tablas de riesgo ${ }^{21}$ con el fin de dividir la predicción a largo y corto de plazo tanto de recidiva como de progresión para cada paciente. La base de este sistema fueron los datos de la Organización Europea para la investigación y el Tratamiento del Cáncer (EORTC), la cual proporcionó información de 2.596 pacientes con tumor TaT1 de 7 ensayos clínicos aleatorizados, a los cuales no se les realizó una segunda RTU y terapia de mantenimiento con Bacilo de Calmette-Guerin (BCG). El sistema de puntuación se basa en los seis factores clínicos y patológicos más significativos:

- Número de tumores

- Tamaño del tumor

- Tasa de recidiva previa

- Categoría T

- Presencia concomitante de CIS

- Grado tumoral

La Tabla 4 ilustra el valor correspondiente a varios factores para calcular la puntuación total tanto de la recidiva como de la progresión. La Tabla 5 muestra la puntuación total estratificada, como en el artículo original ${ }^{21}$, en cuatro categorías que reflejan varias probabilidades de recidiva y progresión al año y a los 5 años.

Con la combinación de dos de las cuatro categorías para la recidiva y la progresión, el grupo de trabajo de la EUA sugiere que se use, tal y como se muestra en el extremo derecho de la Tabla 5, en un sistema que defina grupo de bajo, intermedio y alto riesgo de progresión y recidiva.

Tabla 3.Recomendaciones para el diagnóstico de los tumores de vejiga no músculo invasivos

- Ecografía renal y vesical, urografía intravenosa (UIV) o uroTAC en determinados casos (tumores localizados en el trígono) (grado de recomendación: B).

- Cistoscopia con descripción del tumor (localización, tamaño, número y apariencia) y anormalidades en la mucosa.

- Citología urinaria.

- Resección transuretral (RTU) en un fragmento para tumores pequeños (menores de $1 \mathrm{~cm}$ ). El fragmento debe incluir la porción inferior de la pared vesical (grado de recomendación: B).

- RTU en fracciones (incluido el tejido muscular) para tumores grandes (grado de recomendación: B).

- Biopsias de urotelio de apariencia anormal, biopsias de mucosa de apariencia normal cuando la citología sea positiva o cuando el tumor exofítico sea de apariencia no papilar (grado de recomendación: C).

- Biopsia de la uretra prostática en el caso de tumores del cuello vesical, cuando se sospeche o esté presente CIS, en el caso de citología positiva sin evidenciar tumor en la vejiga o cuando existen anormalidades visibles a nivel de la uretra prostática. Si no se ha realizado durante la resección inicial, puede llevarse a cabo durante la segunda resección. La biopsia debe tomarse usando el asa del resector en el área precolicular (grado de recomendación: C).

- Si disponemos de material, biopsia guiada por fluorescencia cuando se sospeche de CIS en vejiga (por ejemplo citología positiva, tumor recurrente con una historia previa de tumor de alto grado) (grado de recomendación: C).

- Una segunda resección a las 2-6 semanas tras la resección inicial cuando esta ha sido incompleta o cuando se diagnostica un $\mathrm{T} 1$ o alto grado (grado de recomendación: B).

- El informe patológico debe especificar el grado, la profundidad de la invasión tumoral, y sí existe lámina propia o muscular en la muestra (grado de recomendación: C). 
Tabla 4. Ponderación usada para calcular la puntuación de recidiva y progresión (cis=carcinoma in situ)

\begin{tabular}{lcc}
\hline Factor & Recidiva & Progresión \\
\hline Número de tumores & 0 & 0 \\
$\quad$ Unico & 3 & 3 \\
$2-7$ & 6 & 3 \\
$\quad 88$ & & \\
Diámetro tumoral & 0 & 0 \\
$\quad<3 \mathrm{~cm}$ & 3 & 3 \\
$\quad \geq 3 \mathrm{~cm}$ & & \\
Tasa de recidiva previa & 0 & 0 \\
$\quad$ Primario & 2 & 2 \\
$\quad \leq 1$ recidiva/año & 4 & 2 \\
$\quad>1$ recidiva/año & & \\
Categoría & 0 & 0 \\
$\quad$ Ta & 1 & 4 \\
$\quad$ T1 & & \\
Cis concomitante & 0 & 0 \\
$\quad$ No & 1 & 6 \\
$\quad$ Si & & \\
Grado (OMS 1973) & 0 & 0 \\
$\quad$ G1 & 1 & 5 \\
G2 & 2 & $0-23$ \\
G3 & $0-17$ & \\
Puntuación total & & \\
\hline
\end{tabular}

\section{Quimioterapia intravesical adyuvante}

7.1. Instilación intravesical única en el postoperatorio inmediato

Los tumores TaT1 recurren con frecuencia y progresan a enfermedad músculo-invasiva en un número limitado de casos. Por lo tanto es necesario que consideremos una terapia adyuvante en todos los pacientes.

En un meta-análisis de siete estudios aleatorizados, demuestra que la instilación inmediata de qui- mioterapia tras RTU disminuye el porcentaje de recidiva en un $12 \%$ y el riesgo de recidiva en un $39 \%$. El beneficio fue confirmado en tumores únicos y en múltiples ${ }^{22}$ (nivel de evidencia: 1a).

La diferencia del $12 \%$ significa que 8,5 pacientes deberían ser tratados para prevenir una recidiva.

El efecto podría explicarse por la destrucción de las células tumorales circulantes o como un efecto ablativo de las células residuales tumorales en el lugar de la resección.

El tiempo de instilación es crucial. En todos los estudios, la instilación se administró entre las primeras 24 horas. Un estudio informó que si la primera instilación no se administraba el mismo día de la RTU, el riesgo relativo de recidiva se incrementaba el doble ${ }^{23}$ (Nivel de evidencia: 2a).

Mitomicina C (MMC), epirrubicina y doxorrubicina han demostrado unos efectos beneficiosos comparables $^{22}$ (nivel de evidencia: $1 \mathrm{~b}$ ).

Las complicaciones graves son muy raras. Se han publicado cuando se ha producido una extravasación de la quimioterapia ${ }^{24}$. Así, la instilación inmediata debería omitirse en caso de apertura o sospecha perforación intra o extraperitoneal, que es más frecuente que aparezcan tras RTU extensas. Se deben de dar instrucciones claras a enfermería con el fin de controlar el flujo libre del catéter vesical al final de la instilación.

\subsection{Instilaciones adicionales de quimioterapia intravesical}

La necesidad de una terapia adyuvante dependerá de los factores pronósticos del paciente. En pacientes

Tabla 5. Probabilidad de recidiva y progresión de acuerdo con una puntuación total (calculadoras electrónicas de las Tablas 4 y 5 están disponibles en www.eortc.be/tools/bladdercalculator/

\begin{tabular}{|c|c|c|c|c|c|}
\hline \multirow[t]{2}{*}{ Puntuación de recidiva } & \multicolumn{2}{|c|}{ Probabilidad de recidiva al año } & \multicolumn{2}{|c|}{ Probabilidad de recidiva 5 años } & \multirow[t]{2}{*}{ Grupo riesgo recidiva } \\
\hline & $\%$ & (95\%IC) & $\%$ & $(95 \% \mathrm{IC})$ & \\
\hline 0 & 15 & $(10-19)$ & 31 & $(24-37)$ & Bajo riesgo \\
\hline $1-4$ & 24 & $(21-26)$ & 46 & $(42-49)$ & Riesgo intermedio \\
\hline $5-9$ & 38 & $(35-41)$ & 62 & (58-65) & Riesgo intermedio \\
\hline $10-17$ & 61 & $(55-67)$ & 78 & $(73-84)$ & Alto riesgo \\
\hline \multirow[t]{2}{*}{ Puntuación de progresión } & \multicolumn{2}{|c|}{ Probabilidad de progresión al año } & \multicolumn{2}{|c|}{ Probabilidad de progresión 5 años } & Grupo riesgo progresión \\
\hline & $\%$ & $(95 \% \mathrm{IC})$ & $\%$ & $(95 \% \mathrm{IC})$ & \\
\hline 0 & 0,2 & $(0-0,7)$ & 0,8 & $(0-1,7)$ & Bajo riesgo \\
\hline $2-6$ & 1 & $(0,4-0,16)$ & 6 & $(5-8)$ & Riesgo intermedio \\
\hline $7-13$ & 5 & $(4-7)$ & 17 & $(14-20)$ & Alto riesgo \\
\hline $14-23$ & 17 & $(10-24)$ & 45 & (35-55) & Alto riesgo \\
\hline
\end{tabular}


con un bajo riesgo de recidiva (Tabla 5), una instilación única e inmediata puede considerarse como suficiente $^{22}$ (nivel de evidencia: 1a). Para otros pacientes, sin embargo, es un tratamiento incompleto y la probabilidad de recidiva y/o progresión es considerable.

El efecto de una instilación inmediata de quimioterapia se produce durante el primer y segundo $a_{n}{ }^{25}$ (nivel de evidencia: $1 b$ ).

La elección entre quimioterapia o inmunoterapia con BCG de manera prolongada dependerá del riesgo que se quiera disminuir: recidiva o progresión. Un meta-análisis que comparaba quimioterapia intravesical con RTU sola demostró que la quimioterapia previene la recidiva pero no la progresión ${ }^{26}$ (nivel de evidencia: 1a). La eficacia de la reducción de la recidiva con quimioterapia intravesical ha sido confirmada por otros dos meta-análisis en tumores primarios $^{27}$ y recurrentes ${ }^{28}$.

Todavía existen controversias a cerca de cuánto tiempo y con qué frecuencia debe de administrarse esta quimioterapia. A partir de revisiones sistemáticas de ensayos clínicos aleatorizados publicados en la literatura, comparando diferentes esquemas de instilación de quimioterapia intravesical, tan solo podemos concluir que el esquema de duración e intensidad ideal permanece sin definir debido a la existencia de datos contradictorios ${ }^{29}$.

\subsection{Optimización de la quimioterapia intravesical}

Se ha demostrado que ajustando el pH urinario, disminuyendo la excreción urinaria, y amortiguando la solución intravesical se reducen las tasas de recidiva $^{31}$ (nivel de evidencia: $\left.1 b\right)$.

La concentración es más importante que la duración del tratamiento ${ }^{31}$ (nivel de evidencia: 1b). En base a estos datos, parece aconsejable disolver el quimioterápico en una sustancia tampón y $\mathrm{pH}$ ótimo además de advertir al paciente que no beba durante la mañana antes de la instilación.

\section{Inmunoterapia intravesical adyuvante con BCG}

Cuatro meta-análisis han confirmado que la BCG tras la RTU es superior a la RTU sola o RTU con quimioterapia en la prevención de la recidiva de tumores TaT1 ${ }^{32-35}$ (Nivel de evidencia: 1a).

Dos meta-análisis han demostrado que el tratamiento con BCG previene, o al menos retrasa, el riesgo de progresión tumoral ${ }^{36-37}$ (nivel de evidencia: 1a). Se han conseguido reducción del $27 \%$ en la odds de progresión con BCG $(\mathrm{p}=0,0001)$. El tamaño de la reducción es similar tanto en pacientes con TaT1 como aquellos que presentan $\mathrm{CIS}^{36}$.

Otros dos meta-análisis, sin embargo, sugieren un posible sesgo a favor de la BCG por la inclusión de pacientes previamente tratados con quimioterapia intravesical ${ }^{38,39}$.

\subsection{Esquema y dosis óptimas de BCG}

Para una óptima eficacia, la BCG debería darse en un esquema de mantenimiento ${ }^{35,37}$ (nivel de evidencia: 1 a). En un meta-análisis de la EORTC, solo los pacientes que recibían terapia de mantenimiento se beneficiaban. En cuatro ensayos donde no se administraba terapia de mantenimiento, no se observó una reducción de la progresión. En los 20 ensayos donde se administró de alguna manera una terapia de mantenimiento, se observó una reducción del 37\% en el riesgo de progresión ( $\mathrm{p}=0,00004)$. El meta-análisis no fue capaz de determinar que esquema de mantenimiento con BCG era el más efectivo $^{36}$. En un meta-análisis concluye que se requiere al menos un año de mantenimiento con BCG para mostrar una superioridad de la BCG sobre Mitomicina $\mathrm{C}$ en la prevención de la recidiva y la progresión ${ }^{35,37}$.

La inducción con instilaciones de BCG se administran clásicamente de acuerdo a un esquema empírico de inducción de 6 semanas de duración y se han utilizado diferentes esquemas de mantenimiento, hasta por encima de las 30 instilaciones durante 3 años $^{40}$. El número óptimo de las instilaciones durante la inducción así como la frecuencia y duración óptima de las instilaciones de mantenimiento permanece sin aclararse. Basado en el grado de la respuesta inmune intravesical, se sugiere que 3 instilaciones semanales consecutivas aportan una máxima respuesta ${ }^{41}$

Para reducir la toxicidad, se propone disminuir a un tercio y un cuarto de la dosis de BCG. Comparando un tercio de la dosis con la dosis total no se han encontrado diferencias en cuanto a la eficacia. Sin embargo, se sugiere que una dosis máxima de BCG puede ser más efectiva en la enfermedad multifocal $^{42}$ (nivel de evidencia: 1b). Aunque pocos pacientes presentan toxicidad con la reducción de la dosis, la incidencia de toxicidad sistémica grave es similar. La reducción de un sexto de la dosis aporta un descenso en la eficacia con igual toxicidad ${ }^{43}$. 


\subsection{Toxicidad de la $B C G$}

Las muertes debidas a sepsis por BCG así como la alta frecuencia de cistitis inducida por BCG comprometen su uso. Sin embargo, con la mayor experiencia en su aplicación, ahora los efectos secundarios son menos severos. Los efectos secundarios graves aparecen en menos del $5 \%$ de los pacientes ${ }^{44}$ (nivel de evidencia: 1b). Las complicaciones mayores pueden aparecer tras una absorción sistémica. Por este motivo no debe de administrarse durante las dos primeras semanas tras la RTU, en pacientes con hematuria o tras un sondaje traumático.

\subsection{Indicaciones para la BCG}

Aunque la BCG es un tratamiento muy efectivo, existe un consenso a la hora de no tratar a todos los pacientes con cáncer vesical no músculo invasivo debido al riesgo de toxicidad. BCG debe ser considerada como un sobretratamiento en tumores de bajo riesgo de recidiva y progresión (Tabla 5). En pacientes con alto riesgo de progresión, después de una instilación inmediata de quimioterapia, está indicando un esquema de mantenimiento con BCG.

Aunque los pacientes de riesgo intermedio de progresión fueron incluidos en un meta-análi$\operatorname{sis}^{35,37}$, no disponemos de datos que muestren una superioridad en el uso de BCG en estos pacientes. Se podría ofrecer terapia con BCG si la quimioterapia es mal tolerada o si el paciente continua recidivando a pesar de las instilaciones repetidas de quimioterapia. Se debería dar BCG al menos durante un año. Las recomendaciones sobre la terapia intravesical se resumen en la Tabla 6 .

\section{Tratamientos tras fracaso de la terapia intravesical}

Los pacientes con recidivas no músculo invasivas tras instilación de quimioterapia pueden beneficiarse de instilaciones con $\mathrm{BCG}^{38}$.

El tratamiento con BCG se considera que ha fallado en las siguientes situaciones:

- Si se diagnostica un tumor músculo invasivo.

- Si un tumor de alto grado está presenta tanto a los 3 como a los 6 meses $^{45}$. En pacientes a los cuales se presenta el tumor a los tres meses una terapia adicional con BCG provoca una respuesta completa en más del 50\% de los $\operatorname{casos}^{45,46}$.

- Cualquier empeoramiento de la enfermedad bajo el tratamiento con BCG a pesar de una respuesta inicial, como mayor número de recidivas, un alto $\mathrm{T}$ o grado, aparición de CIS (nivel de evidencia: 3).

Pacientes con una recidiva tardía tras completar una terapia con BCG pueden tratarse de acuerdo a su riesgo (Tablas 4 y 5).

Los cambios de BCG a quimioterapia intravesical o dispositivos de asistencia para la instilación intravesical pueden responder en casos seleccionados en los que ha fallado la BCG. Sin embargo las experiencias con estos son limitadas y estas estrategias deben de considerarse como experimentales. Dado el alto riesgo de desarrollo de tumor músculo invasivo en estos pacientes ${ }^{45,47}$ (nivel de evidencia 3) se aboga fuertemente por la realización de una cistectomía inmediata.

\section{Cistectomía}

Muchos expertos consideran que es razonable proponer una cistectomía inmediata en aquellos

Tabla 6. Recomendaciones para la terapia adyuvante

- El tipo de terapia intravesical se basa en grupos de riesgo (Tabla 5).

- En pacientes con bajo riesgo de recidiva y progresión, una instilación de quimioterapia inmediata está altamente recomendada como complementa terapia adyuvante (grado de recomendación: A).

- En pacientes con un riesgo intermedio o alto de recidiva o un riesgo intermedio de progresión, una instilación inmediata de quimioterapia debería seguirse además de instilaciones de quimioterapia o un mínimo de un año con bacilo de Calmette-Guerin (grado de recomendación: A)

- Si usamos quimioterapia, debemos de advertir que debe usarse con un óptimo pH y para mantener las concentraciones durante la instilación mediante la reducción de la toma de líquidos. El esquema óptimo y la duración de la quimioterapia permanece sin aclarar, pero probablemente se debe administrar durante 6-12 meses (grado de recomendación: B).

- En pacientes con alto riesgo de progresión, después de una instilación inmediata de quimioterapia, se usara BCG intravesical al menos durante 1 año (grado de recomendación: A).

- La cistectomía inmediata debe ofrecerse a los pacientes con alto riesgo de progresión tumoral. En pacientes en los que ha fallado la BCG se recomienda la cistectomía (grado de recomendación: C). 
Tabla 7. Recomendaciones para el seguimiento mediante cistoscopias

- Pacientes con tumores de bajo riesgo de recidiva y progresión deberían realizarse una cistoscopia a los 3 meses. Si esta es negativa, la siguiente sería a los 9 meses y posteriormente anual durante 5 años (grado de recomendación: C).

- Pacientes con tumores de alto riesgo de progresión deberían realizarse una cistoscopia y una citología urinaria a los tres meses. Si es negativa, las siguientes cistoscopias y citologías deberían repetirse cada tres meses durante un periodo de 2 años, cada 4 meses en el tercer año, cada 6 meses hasta los 5 años, y después anualmente. Se recomienda una exploración anual del tracto urinario inferior (grado de recomendación: $\mathrm{C}$ ).

- Pacientes con riesgo intermedio de recidiva y/o progresión deberían llevar un esquema de seguimiento usando cistoscopia y citología adaptado de acuerdo a factores individuales (grado de recomendación: C).

pacientes que tienen riesgo de progresión. De acuerdo a las tablas de riesgo de la EORTC (Tablas 4 y 5) estos son:

- Múltiples recidivas de tumores de alto grado.

- Tumores de alto grado T1

- Tumores de alto grado con CIS concomitante

Se recomienda realizar una cistectomía en pacientes en los cuales ha fallado la BCG. Retrasarla puede llevar a un descenso en la supervivencia específica de la enfermedad ${ }^{48}$.

\section{Seguimiento}

Dado el riesgo de recidiva y de progresión los pacientes necesitan un seguimiento. Sin embargo, la frecuencia y duración de las cistoscopias así como las pruebas correspondientes al tracto urinario superior deben de reflejar el grado de riesgo ${ }^{21}$.

- La detección de una invasión muscular o de una recidiva de un tumor no músculo invasivo de alto grado es crítica, y un retraso en el diagnóstico o en la terapia puede amenazar la vida del paciente.

- La recidiva de tumores en el grupo de bajo riesgo siempre suele ser de bajo grado y estadio. Recidivas pequeñas, no invasivas (Ta), de bajo grado y papilares no representan un riesgo inmediato para el paciente y la detección temprana no es esencial para una terapia exitosa ${ }^{49}$ (nivel de evidencia: $2 b$ ).

- El resultado de la primera cistoscopia a los 3 meses de la RTU tiene factor pronóstico tanto para la recidiva como para la progresión ${ }^{21,47,50}$ (nivel de evidencia: 1a). La primera cistoscopia se debería de realizar siempre a los 3 meses de haber realizado la RTU.

- El riesgo de recidiva a nivel del tracto urinario inferior se incrementa en pacientes con tumores múltiples de alto riesgo ${ }^{9}$ (nivel de evidencia: 3 ).

Las recomendaciones para un esquema de seguimiento se encuentran en la Tabla 7.

\section{REFERENCIAS}

1. Oosterlinck W, Lobel B, Jakse G, Malmström PU, Stöckle M, Sternberg C; European Association of Urology (EAU) Working Group on Oncological Urology, Guidelines on bladder cancer. Eur Urol 2002;41(2):105-112.

2. Ferlay J, Bray F, Pisani P, Parkin DM. Globcan 2002, Cancer Incidence, Mortality and Prevalence Worldwide, IARC Cancer Base No. 5, version 2.0. Lyon: IARCC Press; 2004.

3. D.H. Sobin and Ch. Wittekind, TNM classification of malignant tumours (6th ed.), Wiley-Liss, New York, USA 2002:199-202.

4. G. Sauter, F. Algaba, M. Amin et al. Tumours of the urinary system: non-invasive urothelial neoplasias. In: J.N. Eble, G. Sauter, Epstein Jl and I. Sesterhenn, Editors, WHO Classification of Tumours of the Urinary System and Male Genital Organs, IARCC Press, Lyon (2004).

5. Bol MG, Baak JP, Buhr-Wildhagen S, Kruse AJ, Kjellevold KH, Janssen EA, Mestad O, Øgreid P. Reproducibility and prognostic variability of grade and lamina propria invasion in stages Ta, T1 urothelial carcinoma of the bladder. J Urol 2003;169(4): 1291-1294.

6. McCahy PJ, Harris CA, Neal DE. The accuracy of recording of occupational history in patients with bladder cancer. Br J Urol 1997;79(1):91-93.

7. Zeegers MP, Tan FE, Dorant E, van den Brandt PA. The impact of characteristics of cigarette smoking on urinary tract cancer risk: a meta-analysis of epidemiologic Studies. Cancer 2000;89 (3):630-639.

8. Palou J, Rodríguez-Rubio F, Huguet J, Segarra J, Ribal MJ, Alcaraz A, Villavicencio H.. Multivariate analysis of clinical parameters of synchronous primary superficial bladder cancer and upper urinary tract tumours. J Urol 2005;174(1):859-861.

9. Millán-Rodríguez F, Chéchile-Toniolo G, Salvador-Bayarri J, Huguet-Pérez J, Vicente-Rodríguez J. Upper urinary tract tumors after primary superficial bladder tumors: prognostic factors and risk groups. J Urol 2000;164(4);1183-1187.

10. Lokeshwar VB, Habuchi T, Grossman HB et al. Bladder tumor markers beyond cytology: international consensus panel on bladder tumor markers. Urology 2005;66 (Suppl 6A):35-63.

11. Raitanen MP, Aine R, Rintala E, Kallio J, Rajala P, Juusela H, Tammela TL; FinnBladder Group. Differences between local and review urinary cytology and diagnosis of bladder cancer. An interobserver multicenter analysis. Eur Urol 2002;41(3): 284-289.

12. Lopez-Beltran A, Bassi P, Pavone-Macaluso M, Montironi R. Handling and pathology reporting of specimens with carcinoma of the urinary bladder, ureter, and renal pelvis. Eur Urol 2004;45(3):257-266.

13. Brausi M, Collette L, Kurth K, van der Meijden AP, Oosterlinck W, Witjes JA, Newling D, Bouffioux C, Sylvester RJ; EORTC GenitoUrinary Tract Cancer Collaborative Group. Variability in the recurrence rate at first follow-up cystoscopy after TUR in stage Ta T1 transitional cell carcinoma of the bladder: a combined analysis of seven EORTC Studies. Eur Urol 2002;41(5): 523-531. 
14. Van der Meijden A, Oosterlinck W, Brausi M, Kurth KH, Sylvester R, de Balincourt C. Significance of bladder biopsies in Ta,T1 bladder tumours: a report of the EORTC Genito-Urinary Tract Cancer Cooperative Group. EORTC-GU Group Superficial Bladder Committee. Eur Urol 1999(4);5:267-271.

15. Matzkin H, Soloway MS, Hardeman S. Transitional cell carcinoma of the prostate. J Urol 1991;146(6);1207-1212.

16. Mungan MU, Canda AE, Tuzel E, Yorukoglu K, Kirkali Z. Risk factors for mucosal prostatic urethral involvement in superficial transitional cell carcinoma of the bladder. Eur Urol 2005;48(5):760-763.

17. Schmidbauer J, Witjes F, Schmeller N, Donat R, Susani M, Marberger M; Hexvix PCB301/01 Study Group., Improved detection of urothelial carcinoma in situ with hexaminolevulinate fluorescence cystoscopy. J Urol 2004:171(1);135-138.

18. Denzinger S, Burger M, Walter B, Knuechel R, Roessler W, Wieland WF, Filbeck T. Clinically relevant risk of reduction in risk of recurrence of superficial bladder cancer using 5-aminolevulinic acid-induced fluorescence diagnosis: 8-year results of prospective randomized study. Urology 2007;69(4):675-679.

19. Jakse G, Algaba F, . Malmström PU, OosterlinckW. A secondlook TUR in T1 transitional cell carcinoma: why?. Eur Urol 2004;45(5):539-546.

20. Divrik RT, Yildirim U, Zorlu F, Ozen H. The effect of repeat transurethral resection on recurrence and progression rates in patients with T1 tumours of the bladder who received intravesical mitomycin: a prospective, randomized clinical trial. J Urol 2006;175(5):1641-1644.

21. Sylvester RJ, van der Meijden AP, Oosterlinck W, Witjes JA, Bouffioux C, Denis L, Newling DW, Kurth K.. Oosterlinck et al. Predicting recurrence and progression in individual patients with stage Ta T1 bladder cancer using EORTC risk tables: a combined analysis of 2596 patients from seven EORTC trials. Eur Urol 2006;49(3):466-477

22. Sylvester RJ, Oosterlinck W, . van der Meijden A., A single immediate postoperative instillation of chemotherapy decreases the risk of recurrence in patients with stage Ta T1 bladder cancer: a meta-analysis of published results of randomized clinical trials. J Urol 2004; 171(6 Pt 1):2186-2190.

23. Kaasinen E, Rintala E, Hellström P, Viitanen J, Juusela H, Rajala P, Korhonen H, Liukkonen T; FinnBladder Group. The FinnBladder Group, Factors explaining recurrence in patients undergoing chemoimmunotherapy regimens for frequently recurring superficial bladder carcinoma. Eur Urol 2002;42(2); 167-174.

24. Oddens JR, van der Meijden AP, Sylvester R..One immediate postoperative instillation of chemotherapy in low risk Ta, T1 bladder cancer patients. Is it always safe?. Eur Urol 2004;46 (3):336-338.

25. Solsona E, Iborra I, Ricos JV, Monros JL, Casanova J, Dumont R. Effectiveness of a single immediate mitomycin C instillation in patients with low risk superficial bladder cancer: short and long-term follow-up. J Urol 1999;161(4):1120-1123.

26. Pawinski A, Sylvester R, Kurth $\mathrm{KH}$, et al. A combined analysis of European Organization for Research and Treatment of Cancer, and Medical Research Council randomized clinical trials for the prophylactic treatment of Ta T1 bladder cancer. European Organization for Research and Treatment of Cancer Genitourinary Tract Cancer Cooperative Group and the Medical Research Council Working Part on Superficial Bladder Cancer. J Urol 1996;156(6):1934-1941.

27. Huncharek M, Geschwind, JF. Witherspoon B. McGarry R, Adcock D, Intravesical chemotherapy prophylaxis in primary superficial bladder cancer: a meta-analysis of 3703 patients from 11 randomized trials. J Clin Epidemiol. 2000;53(7):676-680.
28. Huncharek M, McGarry R, Kupelnick,B. Impact of intravesical chemotherapy on recurrence rate of recurrent superficial transitional cell carcinoma of the bladder: results of a meta-analysis. Anticancer Res 2001;21(1B):765-770.

29. Sylvester RJ, Oosterlinck W, Witjes JA. The schedule and duration of intravesical chemotherapy in patients with non-muscle-invasive bladder cancer: a systematic review of the published results of randomized clinical trials. Eur Urol 2008;53(4):709-719.

30. Au JL, Badalament RA, Wientjes MG, Young DC, Warner JA, Venema PL, Pollifrone DL, Harbrecht JD, Chin JL, Lerner SP, Miles BJ; International Mitomycin C Consortium. Methods to improve efficacy of intravesical mitomycin C: results of a randomized phase III trial. J Natl Cancer Inst 2001:93(8);597-604.

31. Kuroda M, Niijima T, KotakeT, Akaza H, Hinotsu S; 6th Trial of the Japanese UrologicalCancer Research Group.. Effect of prophylactic treatment with intravesical epirubicin on recurrence of superficial bladder cancer-The 6th Trial of the Japanese Urological Cancer Research Group (JUCRG): a randomized trial of intravesical epirubicin at dose of $20 \mathrm{mg} / 40 \mathrm{ml}$, $30 \mathrm{mg} / 40 \mathrm{ml}, 40 \mathrm{mg} / 40 \mathrm{ml}$. Eur Urol 2004; 45:600-605.

32. Shelley MD, Kynaston H, Court J, Wilt TJ, Coles B, Burgon K, Mason MD. A systematic review of intravesical bacillus Calmette-Guérin plus transurethral resection versus transurethral resection alone in $\mathrm{Ta}$ and $\mathrm{T} 1$ bladder cancer. $\mathrm{BJU}$ International 2001;88(3):209-216.

33. Han RF, Pan JG. Can intravesical bacillus Calmette-Guérin reduce recurrence in patients with superficial bladder cancer? A metaanalysis of randomized trials. Urology 2006;67(6): 1216-1223.

34. Shelley MD, Wilt TJ, Court J, Coles B, Kynaston H, Mason MD.. Intravesical bacillus Calmette-Guerin is superior to mitomycin $\mathrm{C}$ in reducing tumour recurrence in high-risk superficial bladder cancer: a meta-analysis of randomized trials. BJU International 2004;93:(4) 485-490.

35. Böhle A, Jocham D, Bock PR, Intravesical bacillus CalmetteGuerin versus mitomycin $\mathrm{C}$ for superficial bladder cancer: a formal meta-analysis of comparative studies on recurrence and toxicity. J Urol 2003;169:(1)90-95.

36. Sylvester R.J, van der Meijden and D.L. Lamm, Intravesical bacillus Calmette-Guerin reduces the risk of progression in patients with superficial bladder cancer: a combined analysis of the published results of randomized clinical trials. J Urol 2002; 168(5):1964-1970.

37. Böhle A, Bock PR. Intravesical bacillus Calmette-Guerin versus mitomycin $\mathrm{C}$ in superficial bladder cancer: formal meta-analysis of comparative studies on tumor progresión. Urology 2004;63(4):682-687.

38. Huncharek M, Kupelnick B. Impact of intravesical chemotherapy versus BCG immunotherapy on recurrence of superficial transitional cell carcinoma of the bladder. Am J Clin Oncol 2003;26(4):402-407.

39. Huncharek M, Kupelnick B, The influence of intravesical therapy on progression of superficial transitional cell carcinoma of the bladder: a meta-analytic comparison of chemotherapy versus bacilli Calmette-Guerin immunotherapy. Am J Clin Oncol 2004;27(5):522-528.

40. Lamm DL, Blumenstein BA, Crissman JD, Montie JE, Gottesman JE, Lowe BA, Sarosdy MF, Bohl RD, Grossman HB, Beck TM, Leimert JT, Crawford ED. Maintenance bacillus Calmette-Guerin immunotherapy for recurrent Ta, T1 and carcinoma in situ transitional cell carcinoma of the bladder: a randomized Southwest Oncology Group Study. J Urol 2000:163(4);1124-1129.

41. Zlotta AR, van Vooren JP, Huygen K, Drowart A, Decock M, Pirson M, Jurion F, Palfliet K, Denis O, Simon J, Schulman CC.What is the optimal regimen for BCG intravesical therapy?. Eur Urol 2000;37(4):470-477. 
42. Martínez-Piñeiro JA, Martínez-Piñeiro L, Solsona E, Rodríguez RH, Gómez JM, Martín MG, Molina JR, Collado AG, Flores N, Isorna S, Pertusa C, Rabadán M, Astobieta A, Camacho JE, Arribas S, Madero R; Club Urológico Español de Tratamiento Oncológico (CUETO). Has a 3-fold decreased dose of bacillus Calmette-Guerin the same efficacy against recurrences and progression of T1G3 and Tis bladder tumours than the standard dose? Results of a prospective randomized trial. J Urol 2005; 174(4Pt1):1242-1247.

43. Ojea A, Nogueira JL, Solsona E, Flores N, Gómez JM, Molina JR, Chantada V, Camacho JE, Piñeiro LM, Rodríguez RH, Isorna S, Blas M, Martínez-Piñeiro JA, Madero R; CUETO Group (Club Urológico Español De Tratamiento Oncológico). A multicentre, randomised prospective trial comparing three intravesical adjuvant therapies for intermediate-risk superficial bladder cancer: low-dose bacillus Calmette-Guerin (27 mg) versus very low-dose bacillus Calmette-Guerin (13.5 mg) versus mitomycin C. Eur Urol 2007;52(5):1398-1406.

44. Van der Meijden AP, Sylvester RJ, Oosterlinck W, Hoeltl W, Bono AV; EORTC Genito-Urinary Tract Cancer Group. Maintenance bacillus Calmette-Guerin for Ta, T1 bladder tumors is not associated with increased toxicity: results from a European Organisation for Research and Treatment of Cancer Genito-Urinary Group Phase III Trial. Eur Urol 2003:44(4);429434.

45. Herr HW, Dalbagni G. Defining bacillus Calmette-Guerin refractory superficial bladder tumours. J Urol. 2003;169:(5): 1706-1708.

46. Sylvester RJ, van der Meijden A, Witjes JA, Jakse G, Nonomura N, Cheng C, Torres A, Watson R, Kurth KH. High grade Ta urothelial carcinoma and carcinoma in situ of the bladder. Urology 2005;66 (6 Suppl 1):90-107.
47. Solsona E, Iborra I, Dumont R, Rubio-Briones J, Casanova J, Almenar $\mathrm{S}$. The 3-month clinical response to intravesical therapy as a predictive factor for progression in patients with high risk superficial bladder cancer. J Urol 2000(3 Pt1);164:685689.

48. Raj GV, Herr H, Serio AM, Donat SM, Bochner BH, Vickers AJ, Dalbagni G.,Treatment paradigm shift may improve survival of patients with high risk superficial bladder cancer. J Urol 2007; 177(4):1283-1286.

49. Gofrit ON, Pode D, Lazar A, Katz R, Shapiro A. Watchful waiting policy in recurrent Ta G1 bladder tumors. Eur Urol 2006; 49:(2)303-307.

50. Holmang S, Johansson SL. Stage Ta-T1 bladder cancer: the relationship between findings at first followup cystoscopy and subsequent recurrence and progresión. J Urol 2002;176:16341637.

Autor para correspondencia. Dr. Marko Babjuk

Department of Urology,

General Teaching Hospital, First Faculty of Medicine,

Charles University, Ke Karlovu 6, Praha 2, 12808 Czech Republic. Tel. +420224967848 Fax: +420224967102.

E-mail address: marko.babjuk@1f1.cuni.cz (M. Babjuk)

Correspondencia del traductor: Dr. Saturnino Luján Marco Servicio de Urología

Hospital Universitario La Fe

Avda. Campanar, 21 - 46009 Valencia

Tel.: 963862700

E-mail autor: slujanmarco@comv.es

Información artículo: Revisión - Cáncer de vejiga 